\title{
Resistencia a múltiples antibióticos en serovariedades de Salmonella aisladas de muestras clínicas y alimentos
}

\author{
Flavia Ortiz ${ }^{1}$ (D) Natalie Weiler $^{1}$ (D) , Mercedes Alvarez ${ }^{1}$ (D) María Verónica Orrego $^{1}$ (D), \\ Aníbal Kawabata1 ${ }^{(i D}$, Esteban Riera2 ${ }^{\text {(D) }}$, Celia López ${ }^{3}$ (D) Liz Acosta ${ }^{4}$ (D) Minako

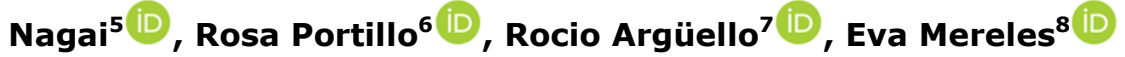 \\ ${ }^{1}$ Ministerio de Salud Pública y Bienestar Social, Laboratorio Central de Salud Pública. \\ Asunción, Paraguay \\ ${ }^{2}$ Laboratorio Riera. Asunción Paraguay \\ ${ }^{3}$ Servicio Nacional de Salud Animal SENACSA. San Lorenzo Paraguay \\ ${ }^{4}$ Hospital Nacional de Itaugua. Itaugua Paraguay \\ ${ }^{5}$ Instituto de Previsión Social/Hospital Central. Asunción Paraguay \\ ${ }^{6}$ Centro médico La Costa. Asunción Paraguay \\ ${ }^{7}$ Hospital General Pediátrico. San Lorenzo Paraguay \\ ${ }^{8}$ Hospital Regional de Ciudad del Este. Ciudad del Este Paraguay
}

Cómo referenciar este artículo/ How to reference this article:

\begin{abstract}
Ortiz F, Weiler $N$, Alvarez $M$, Orrego MV, Kawabata A, Riera E, López C, Acosta L, Nagai M, Portillo R, Argüiello R, Mereles E. Resistencia a múltiples antibióticos en serovariedades de salmonella aisladas de muestras clínicas y alimentos. Mem. Inst. Investig. Cienc. Salud. 2021; 19(1): 37-47
\end{abstract}

\section{RE S U M E N}

Salmonella enterica es un patógeno transmitido por alimentos y agente etiológico de brotes alimentarios de gran impacto en la salud humana. El aumento de la resistencia bacteriana constituye una amenaza a la salud pública, la aparición de cepas de Salmonella con resistencia a múltiples antimicrobianos (MDR) fue descrita en humanos, alimentos y animales para consumo; por ello se considera muy importante conocer la situación epidemiológica local. El objetivo de este trabajo fue generar información sobre los serotipos circulantes, resistencia a los antibióticos y presencia de resistencia simultánea a múltiples fármacos en Salmonella provenientes de muestras clínicas humanas y muestras de alimentos en el periodo desde 2017 a 2019. Fueron analizadas un total de 668 cepas de Salmonella aisladas en los años 2017, 2018 y 2019 a partir de muestras clínicas humanas y de alimentos, en el Laboratorio Central de Salud Pública y/o remitidas por Laboratorios de la Red de Enteropatógenos. Se observaron serotipos muy diversos con prevalencia del serovar Heidelberg en alimentos y Typhimurium en muestras de humanos. Se encontró que el $45,4 \%$ de las cepas fueron sensibles a todos los antibióticos (ATB), el 35,6\% fueron resistentes de 1 a 6 ATB y el $19 \%$ con sensibilidad intermedia; observándose mayor resistencia a Tetraciclina, Ác. Nalidíxico, Ampicilina y Nitrofurantoína, en menor grado se evidenció resistencia a cefalosporinas $\left(\mathrm{C} 3^{\mathrm{a}} \mathrm{G}\right)$ y a ciprofloxacina. El $16.9 \%$ de las cepas presentaron resistencia múltiple ( 3 o más antibióticos) con 37 fenotipos distintos. Las serovariedades que presentaron mayor resistencia a los antimicrobianos fueron Heidelberg, Schwarzengrund y Typhimurium.

Palabras clave: Salmonella, resistencia, serovar, antimicrobiano.

Fecha de recepción: Diciembre 2020. Fecha de aceptación: Abril 2021

*Autor correspondiente: Flavia Helena Ortiz Arce. Laboratorio Central de Salud Pública, Paraguay.

Teléfono: +595981232923

Email: flavia.arce@gmail.com 


\title{
Resistance to multiple antibiotics in serovarities of Salmonella isolated from clinical samples and foods
}

\begin{abstract}
Salmonella enterica is a foodborne pathogen and etiological agent of food outbreaks with a great impact on human health. The increase in bacterial resistance constitutes a threat to public health. The appearance of Salmonella strains with resistance to multiple antimicrobials (MDR) has already been described in humans, food and animals for consumption; for this reason, it is considered very important to know the local epidemiological situation. The target of this work was to generate information on circulating serotypes, antibiotic resistance and the presence of simultaneous resistance to multiple drugs in Salmonella from human clinical samples and food samples in the period from 2017 to 2019.

A total of 668 Salmonella strains isolated in the years 2017, 2018 and 2019 were analyzed from human and food clinical samples, at the Central Public Health Laboratory and / or sent by Laboratories of the Enteropathogens Network. Very diverse serotypes were observed with prevalence of Heidelberg serovar in food and Typhimurium in human samples. It was found that $45,4 \%$ of the strains were sensitive to all antibiotics (ATB), 35,6\% were resistant from 1 to 6 ATB and $19 \%$ with intermediate sensitivity; observing greater resistance to Tetracycline, Ác. Nalidixic, Ampicillin and Nitrofurantoin, to a lesser degree resistance to cephalosporins $\left(C 3^{a} G\right)$ and ciprofloxacin was evidenced. The $16.9 \%$ the strains presented multiple resistance (3 or more antibiotics) with 37 different phenotypes. The serovars with the highest antimicrobial resistance were Heidelberg, Schwarzengrund and Typhimurium.
\end{abstract}

Keywords: Salmonella, resistance, serovar, antimicrobial.

\section{INTRODUCCIÓN}

Salmonella enterica es uno de los patógenos zoonóticos transmitidos por alimentos de mayor relevancia en el mundo, es una de las principales causas de enfermedades diarreicas en el ser humano, considerada como importante agente etiológico de los brotes de enfermedades transmitidas por alimentos (ETA); puede atravesar toda la cadena alimentaria y es un microorganismo que también ha desarrollado resistencia a los antibióticos, por ello tiene un gran impacto en la salud pública (WHO 2015 ${ }^{(1)}$, CDC $\left.2018^{(2)}\right)$, sanidad animal y en la industria alimentaria debido a la creciente aparición y diseminación de variantes resistentes a múltiples antimicrobianos ${ }^{(3,4)}$.

Las bacterias del género Salmonella se agrupan en dos especies $S$. enterica y $S$. bongori la primera se subdivide en seis subespecies: I enterica, II salamae, IIIa arizonae, IIIb diarizonae, IV houtenae, VI indica; el potencial patogénico para el ser humano está representado por miembros de la subespecie enterica (I) y sus más de 2.500 serotipos $^{(5)}$ que también producen infecciones en animales de sangre caliente, se transmiten por la ruta fecal-oral directamente o a través de alimentos.

Salmonella se encuentra ampliamente distribuida en la naturaleza en el tracto gastrointestinal de mamíferos, aves y reptiles tanto silvestres como domésticos, constituyen su reservorio principal y tienen un papel importante en la diseminación en el medioambiente, a excepción de Salmonella serovar Typhi y Paratyphi cuyo único reservorio es el hombre; se considera que los alimentos son la principal fuente de exposición humana, y se estima que la gran mayoría de las infecciones están asociadas con alimentos de origen animal ${ }^{(6)}$.

La manifestación clínica más característica de salmonelosis en el ser humano es la gastroenteritis que generalmente cursa con cuadros leves y autolimitados, aunque también se observan cuadros invasivos extraintestinales como bacteriemias e infecciones localizadas. El tratamiento de la gastroenteritis va dirigido a la prevención de la deshidratación con reposición de líquidos y electrolitos ${ }^{(7)}$, la terapia antimicrobiana empírica no se recomienda para los casos leves y moderados en personas sanas precisamente para no seleccionar cepas resistentes y solo se debería aplicar a grupos de riesgo o en infecciones extraintestinales ${ }^{(8)}$. En los animales es común la colonización intestinal pero también puede causar enfermedad, la infección 
es de transmisión fecal-oral semejante a la del hombre, ocasiona gastroenteritis y septicemia mortal, particularmente en animales jóvenes ${ }^{(9)}$.

La tolerancia al calor, persistencia a la desecación ${ }^{(5)}$ y capacidad de formar biopelículas sobre diferentes superficies le permitiría a Salmonella sobrevivir a largo plazo en el entorno de las granjas de aves de corral y contaminar la carne y huevos o alimentos derivados, vehículos principales de los brotes de salmonelosis de origen alimentario $^{(10)}$ a esta última propiedad también se atribuye ser un reservorio de bacterias y el aumento de riesgo de contaminación de los alimentos procesados a través de las diferentes superficies durante la cadena productiva, aumentando el potencial de transmisión hasta los consumidores finales ${ }^{(11)}$.

La resistencia antimicrobiana constituye un problema globalizado y creciente, las infecciones causadas por bacterias resistentes se relacionan directamente con mayor morbilidad, mortalidad y gasto hospitalario. En un estudio de revisión de Juan Ignacio Alós $2015^{(12)}$ se visualiza la concepción de la resistencia en diferentes escenarios y no exclusivamente hospitalarios, se describe el vertido de antibióticos al ambiente a través de aguas residuales donde logran coexistir bacterias ambientales, humanas y animales; el intestino humano y animal constituye otro nicho donde puede ocurrir intercambio de genes inter e intraespecies con selección de cepas resistentes; además los antimicrobianos se han usado a dosis bajas y masivamente como promotores de crecimiento animal en la producción pecuaria, también como profilácticos en acuicultura y agricultura contribuyendo a la extensión de la resistencia.

Es una realidad que el uso y abuso de antimicrobianos prescritos en medicina humana y animal ha facilitado la selección y dispersión de patógenos resistentes y en un mundo cada vez más interconectado su propagación no encuentra fronteras.

En su reporte de vigilancia global sobre resistencia a antimicrobianos ${ }^{(13)}$, la Organización Mundial de la Salud (OMS) ha señalado altas tasas de Resistencia en bacterias comunes en todas sus regiones, con el hallazgo de aumento de Resistencia a Cefalosporinas de tercera generación ( $\mathrm{C}^{\mathrm{a}} \mathrm{G}$ ) y Fluoroquinolonas, así como el aumento de bacterias resistentes a carbapenemes, y en los últimos años la OPS/OMS ha emitido alertas epidemiológicas de aumento de resistencia plasmídica a la colistina ${ }^{(14)}$ y resistencia extendida en Salmonella enterica serovar Typhi ${ }^{(15)}$.

El aumento de la resistencia bacteriana supone una amenaza creciente para la salud humana ${ }^{(16)}$, la aparición de cepas de Salmonella con resistencia a múltiples antibióticos (MDR) en diferentes regiones geográficas ya fue descrita en varios estudios que involucran a humanos, alimentos y animales para consumo (17-19), convirtiéndose en un problema globalizado y con alta repercusión económica ${ }^{(20-23)}$; por ello se considera muy oportuno conocer la situación epidemiológica de Salmonella a nivel nacional, y su comportamiento frente a los antibióticos empleados tanto en el sector sanitario como el productivo .

El objetivo de este trabajo fue generar información sobre la prevalencia de serotipos circulantes, resistencia a los antibióticos y presencia de resistencia simultánea a múltiples fármacos en aislados de Salmonella provenientes de muestras clínicas humanas y muestras de alimentos en el periodo desde 2017 a 2019.

\section{MATERIALES Y MÉTODOS.}

Fueron analizadas 668 cepas de Salmonella aisladas en los años 2017, 2018 y 2019 a partir de muestras clínicas humanas y de alimentos, las cuales fueron aisladas en el Laboratorio Central de Salud Pública y/o remitidas por Laboratorios de la Red de Enteropatógenos. Las muestras de alimentos fueron en su mayoría de origen animal (aviar, bovino, porcino, lácteos, balanceados para animales y otros) mientras que las muestras clínicas humanas fueron: heces, sangre, orina y otras secreciones.

Todas las cepas fueron identificadas mediante pruebas metabólicas: TSI, LIA, Sorbitol, Sacarosa, Dulcitol, Malonato y ONPG, y sometidas a pruebas de susceptibilidad antimicrobiana para Ampicilina 10 ug (AMP), Amoxicilina-ácido clavulánico 20/10 ug (AMC), Cefixima 5 ug (CFM), Cefotaxima 30 ug. (CTX), Ácido nalídixico 30 ug (NAL), Ciprofloxacina 5 ug (CIP), Cloranfenicol 30 ug (CHL), Gentamicina 10 ug. (GEN), Nitrofurantoína 300 ug (NIT), Tetraciclina 30 ug. (TCY), y Trimetoprim-Sulfametoxasol $0.125 / 23.75$ ug. (SXT) por difusión en agar (método de 
Kirby-Bauer) siguiendo las normativas de CLSI (Clinical and Laboratory Standards Institute). Se categorizaron como cepas MDR (resistentes a múltiples fármacos) a aquellas que presentaron resistencia al menos a un antibiótico en tres o más grupos o familias, considerando todos los antibióticos testados; con el propósito de tener un amplio panorama de la resistencia a los agentes antimicrobianos (vigilancia RAM) ${ }^{(24-}$ 28). La serovariedad se determinó por aglutinación en láminas utilizando antisueros somáticos y flagelares según el esquema de Kauffmann-White, todos los datos obtenidos fueron recopilados en una base de datos en formato Microsoft Excel. Los resultados y análisis estadísticos realizados se expresaron en proporciones o porcentajes.

\section{RESULTADOS}

De las 668 cepas de Salmonella 53,4 \% fueron procedentes de muestras clínicas humanas y $46,6 \%$ de muestras de alimentos; se encontró gran diversidad de serotipos, un total de 53 serovariedades distintas (Tabla 1): 30 en la población humana predominando Salmonella enterica ssp enterica serovar Typhimurium, Infantis, Newport, Schwarzengrund y 39 serotipos en cepas de alimentos con predominio de Salmonella enterica ssp. enterica serovar Heidelberg, Havana y Oranienburg; en el $14,4 \%$ de todas las cepas estudiadas $(96 / 668)$ no se logró completar la fórmula antigénica de serotipificacion y fueron catalogadas como Salmonella enterica ssp enterica.

Tabla 1: Prevalencia de serotipos de Salmonella aisladas de muestras de alimentos y humanos 2017-2018-2019 - Paraguay

\begin{tabular}{|c|c|c|c|}
\hline $\begin{array}{l}\text { Fuente de } \\
\text { origen }\end{array}$ & $\begin{array}{c}\mathbf{N}^{\circ} \\
\text { aislamientos } \\
(\%)\end{array}$ & Serotipos ( $N^{\circ}$ de aislamientos) & $\begin{array}{c}\mathbf{N}^{\circ} \mathrm{de} \\
\text { serotipos }\end{array}$ \\
\hline \multirow[t]{2}{*}{ Humano } & $357(53,4)$ & $\begin{array}{l}\text { Typhimurium(109),S. enterica ssp enterica(56)", } \\
\text { Infantis(30), Newport(25), Schwarzengrund(21), } \\
\text { Javiana(15), Saintpaul(14), Oranienburg(13), } \\
\text { Enteritidis(10), Poona(9), Braenderup(8), Sandiego(8), } \\
\text { Corvallis(7), Dublin(5), Livingstone(2), Agona(2), }\end{array}$ & \\
\hline & & $\begin{array}{l}\text { Bredeney(2), Anatum(2), Albany(2), Hadar(2), } \\
\text { Westhampton(2), Heidelberg(1), Cerro(1), Mbandaka(1), } \\
\text { Give(1), Molade(1), Rissen(1), Arechavaleta(1), } \\
\text { Panamá(1), Paratyphi B(1), Rubislaw(1), S.enterica ssp } \\
\text { diarizonae(1), S. enterica ssp houtenae(1), S. enterica } \\
\text { ssp salamae(1). }\end{array}$ & 30 \\
\hline Alimento & $311(46,6)$ & $\begin{array}{l}\text { Heidelberg(62), Havana(44), S. enterica ssp. } \\
\text { enterica(40)*, Oranienburg(18),Typhimurium(13), } \\
\text { Schwarzengrund(13), Livingstone(13),Cerro(12), } \\
\text { Braenderup(11), Mbandaka(11), Agona(9), } \\
\text { Montevideo(5)Senftenberg(5), Tennessee(5), Give(4), } \\
\text { Yoruba(4), Newport(3), Bredeney(3), Molade(3), }\end{array}$ & \\
\hline & & $\begin{array}{l}\text { Rideau(3), Soerenga(3), Carrau(2), London(2), } \\
\text { Minnesota(2), Thies(2), Zaiman(2), Javiana(1), } \\
\text { Enteritidis(1), Corvallis(1), Anatum(1), Rissen(1), } \\
\text { S.enterica ssp diarizonae(1), Alminko(1), Bareilly(1), } \\
\text { Cholerasuis(1), Dallgow(1), Ghana(1), Give (1), } \\
\text { Grumpensis(1), Idikan(1), Lexington(1), Soerenga (1), } \\
\text { Tokoin(1). }\end{array}$ & 39 \\
\hline Total & $668(100)$ & & 53 \\
\hline
\end{tabular}

* Aislamientos de Salmonella enterica ssp enterica con fórmula antigénica incompleta (sin serotipo).

Un $45,4 \%$ de los aislamientos (303/668) fueron sensibles a todos los antibióticos testados y $35,6 \%(238 / 668)$ presentaron resistencia desde uno a seis antibióticos; se observó sensibilidad intermedia en 19,0\% (127/668). En general la resistencia antimicrobiana más elevada fue para Tetraciclina, Ac. Nalidíxico y Ampicilina, con ligeras variaciones en tendencia al considerar cada grupo en forma individual (Tabla $2)$; es importante mencionar que en el $27,5 \%$ de las cepas estudiadas presentó sensibilidad intermedia a ciprofloxacina. 
Tabla 2: Resistencia en Salmonella spp. aisladas de muestras de alimentos y humanos 2017-2018-2019 - Paraguay.

\begin{tabular}{|c|c|c|c|}
\hline \multirow[t]{2}{*}{ Antibióticos } & \multicolumn{3}{|c|}{ Número de aislamientos resistentes (\%) } \\
\hline & Alimento $(n=311)$ & Humano $(n=357)$ & Total $(n=668)$ \\
\hline $\begin{array}{l}\boldsymbol{\beta} \text {-lactámicos } \\
\text { Ampicilina (AMP) }\end{array}$ & $34(10,9)$ & $77(21,6)$ & $111(16,6)$ \\
\hline Amoxilina/Ac. Clavulánico (AMC) & $25(8,0)$ & $6(1,7)$ & $31(4,6)$ \\
\hline Cefixime (CFM) & $29(9,3)$ & $24(6,7)$ & $53(7,9)$ \\
\hline Cefotaxima (CTX) & $29(9,3)$ & $24(6,7)$ & $53(7,9)$ \\
\hline \multicolumn{4}{|l|}{ Quinolonas } \\
\hline Ac. Nalidixico (NAL) & $90(28,9)$ & $36(10,1)$ & $126(18,9)$ \\
\hline Ciprofloxacina (CIP) & $18(5,8)$ & $11(3,1)$ & $29(4,3)$ \\
\hline $\begin{array}{l}\text { Otros } \\
\text { Cloranfencol (CHL) }\end{array}$ & $3(1,0)$ & $22(6,2)$ & $25(3,7)$ \\
\hline Gentamicina (GEN) & $2(0,6)$ & $15(4,2)$ & $17(2,5)$ \\
\hline Nitrofurantoína (NIT) & $47(15,1)$ & $40(11,2)$ & $87(13)$ \\
\hline Tetraciclina (TET) & $88(28,3)$ & $86(24,1)$ & $174(26)$ \\
\hline Trimetoprim/Sulfametoxazol (SXT) & $8(2,6)$ & $25(7,0)$ & $33(4,9)$ \\
\hline
\end{tabular}

Los serotipos de Salmonella asociados a mayor resistencia antimicrobiana incluyendo a los antibióticos para tratamiento de infecciones humanas fueron Heidelberg, Schwarzengrund y Typhimurium (Tabla 3).

Tabla 3: Resistencia a los antibióticos en serovariedades prevalentes de Salmonella, aislados a partir de muestras de alimentos y de humanos 2017-2018-2019-Paraguay

\begin{tabular}{|c|c|c|c|c|c|c|c|c|c|}
\hline \multirow{2}{*}{ Antibióticos } & \multicolumn{9}{|c|}{ Serovariedades - Número de aislamientos resistentes (\%) } \\
\hline & $\begin{array}{c}\text { Typhimurium } \\
(n=122)\end{array}$ & $\begin{array}{c}\text { Heidelberg } \\
(n=63)\end{array}$ & $\begin{array}{c}\text { Havana } \\
(n=44)\end{array}$ & $\begin{array}{l}\text { Schwarzengrund } \\
(\mathrm{n}=34)\end{array}$ & $\begin{array}{c}\text { Oranienburg } \\
(n=31)\end{array}$ & $\begin{array}{c}\text { Infantis } \\
(n=30)\end{array}$ & $\begin{array}{c}\text { Newport } \\
(n=28)\end{array}$ & $\begin{array}{c}\text { Braenderup } \\
(n=19)\end{array}$ & $\begin{array}{c}\text { Otros } \\
(n=297)\end{array}$ \\
\hline \multicolumn{10}{|l|}{ ß-lactámicos } \\
\hline AMP & $40(32,8)$ & $21(33,3)$ & $\begin{array}{c}1 \\
(2,3)\end{array}$ & $19(55,9)$ & $2(6,5)$ & $9(30)$ & $2(7,1)$ & $0(0)$ & $\begin{array}{c}17 \\
(5,7)\end{array}$ \\
\hline AMC & $3(2,5)$ & $19(30,2)$ & $\begin{array}{c}1 \\
(2,3)\end{array}$ & $2(5,9)$ & $0(0)$ & $0(0)$ & $1(3,6)$ & $0(0)$ & $5(1,7)$ \\
\hline CFM & $4(3,3)$ & $21(33,3)$ & $0(0)$ & $18(52,9)$ & $2(6,5)$ & $0(0)$ & $0(0)$ & $0(0)$ & $8(2,7)$ \\
\hline CTX & $4(3,3)$ & $21(33,3)$ & $0(0)$ & $18(52,9)$ & $2(6,5)$ & $0(0)$ & $0(0)$ & $0(0)$ & $8(2,7)$ \\
\hline \multicolumn{10}{|l|}{ Quinolonas } \\
\hline NAL & $15(12,3)$ & $62(98,4)$ & $0(0)$ & $13(38,2)$ & $0(0)$ & $0(0)$ & $2(7,1)$ & $1(5,3)$ & $\begin{array}{c}33 \\
(11,1)\end{array}$ \\
\hline CIP & $8(6,6)$ & $13(20,6)$ & $0(0)$ & $0(0)$ & $0(0)$ & $0(0)$ & $0(0)$ & $0(0)$ & $8(2,7)$ \\
\hline \multicolumn{10}{|l|}{ Otros } \\
\hline $\mathrm{CHL}$ & $11(9)$ & $1(1,6)$ & $0(0)$ & $0(0)$ & $1(3,2)$ & $9(30)$ & $1(3,6)$ & $0(0)$ & $2(0,7)$ \\
\hline GEN & $15(12,3)$ & $2(3,2)$ & $0(0)$ & $0(0)$ & $0(0)$ & $0(0)$ & $0(0)$ & $0(0)$ & $0(0)$ \\
\hline NIT & $11(9)$ & $15(23,8)$ & $5(11,4)$ & $2(5,9)$ & $6(19,4)$ & $8(26,7)$ & $1(3,6)$ & $0(0)$ & $\begin{array}{c}39 \\
(13,1)\end{array}$ \\
\hline TET & $49(40,2)$ & $59(93,7)$ & $0(0)$ & $19(55,9)$ & $1(3,2)$ & $9(30)$ & $4(14,3)$ & $0(0)$ & $\begin{array}{c}33 \\
(11,1)\end{array}$ \\
\hline SXT & $6(4,9)$ & $2(3,2)$ & $0(0)$ & $18(52,9)$ & $2(6,5)$ & $0(0)$ & $1(3,6)$ & $0(0)$ & $4(1,3)$ \\
\hline
\end{tabular}

Se observaron fenotipos compatibles con Betalactamasas de espectro extendido (BLEE) y AmpC plasmídico en Schwarzengrund y Heidelberg respectivamente, mecanismos que confieren resistencia a Cefalosporinas ${ }^{2} 3^{a}$ G. 
La resistencia a múltiples fármacos estuvo presente en cada uno de los tres años del periodo de estudio, el $16.9 \%$ de todas las cepas (113/668) fueron resistentes a tres o más antibióticos y categorizadas como MDR (Tabla 4), se observó poca variación al considerar en forma independiente las fuentes de origen: en cepas aisladas de alimentos el $17,7 \%$ fueron MDR y en cepas provenientes de muestras clínicas humanas el 16,2\%.

Tabla 4: Salmonella spp. Resistentes a múltiples antibióticos (MDR), aisladas de alimentos y humanos - Paraguay

\begin{tabular}{cccc}
\hline $\begin{array}{c}\mathbf{N}^{\circ} \text { de Antibióticos } \\
\text { resistentes }\end{array}$ & \multicolumn{4}{c}{ Número de aislamientos resistentes (\%) } \\
\hline & $\begin{array}{c}\text { Alimento } \\
(\mathbf{n = 3 1 1 )}\end{array}$ & $\begin{array}{c}\text { Humano } \\
\mathbf{( n = 3 5 7 )}\end{array}$ & $\begin{array}{c}\text { Total } \\
(\mathbf{n = 6 6 8 )}\end{array}$ \\
\hline 0 & $124(40,0)$ & $179(50,1)$ & $303(45,4)$ \\
$1-2$ & $60(19,3)$ & $65(18,2)$ & $125(18,7)$ \\
$3-4$ & $26(8,4)$ & $40(11,2)$ & $66(9,9)$ \\
$5-6$ & $29(9,3)$ & $18(5,0)$ & $47(7,0)$ \\
$\mathrm{I} \geq 1$ & $72(23,2)$ & $55(15,4)$ & $127(19,0)$ \\
$\mathrm{R} \geq 1$ & $115(37,0)$ & $123(34,5)$ & $238(35,6)$ \\
MDR $\geq \mathbf{3}$ & $\mathbf{5 5 ( \mathbf { 1 7 } , 7 )}$ & $\mathbf{5 8 ( 1 6 , 2 )}$ & $\mathbf{1 1 3 ( 1 6 , 9 )}$ \\
\hline
\end{tabular}

* I: Sensibilidad intermedia **MDR: Resistencia por lo menos a 1 Antibiótico por familia y con diferente espectro de acción. ***En el caso de las Quinolonas la resistencia simultánea a NAL( $1^{\circ}$ Generación) y CIP ( $2^{\circ}$ Generación), se considera como una sola.

Se encontraron 11 serovariedades de Salmonella que presentaron 37 patrones con fenotipos diferentes de resistencia múltiple, 27 en humanos y 13 en alimentos (Tabla $5)$.

Tabla 5: Relación entre patrones de multirresistencia, serovariedades de Salmonella MDR y la fuente de origen. Año 2017-2018-2019.

\begin{tabular}{|c|c|c|c|c|c|}
\hline Patrones MDR & $\begin{array}{l}\mathrm{N}^{\circ} \text { de ATB } \\
\text { resistentes }\end{array}$ & $\begin{array}{c}\text { Fenotipos de } \\
\text { Resistencia }\end{array}$ & $\begin{array}{c}\text { Salmonella } \\
\text { No aislamientos }\end{array}$ & $\begin{array}{c}\text { Serovariedad } \\
\left(n^{\circ}\right)\end{array}$ & $\begin{array}{c}\text { Tipo de muestra } \\
\left(n^{\circ}\right)\end{array}$ \\
\hline P1 & 3 & NAL-NIT-TET & 16 & $\begin{array}{l}\text { Heidelberg (9) } \\
\text { S. enterica ( } 7)\end{array}$ & $\begin{array}{l}\text { Pollo (14) }{ }^{A} \\
\text { Bovino (1) }\end{array}$ \\
\hline P2 & 5 & $\begin{array}{c}\text { NAL-AMC- } \\
\text { AMP-CFM/CTX- } \\
\text { TET }\end{array}$ & 14 & $\begin{array}{l}\text { Heidelberg (13) } \\
\text { S. enterica (1) }\end{array}$ & Pollo (14) \\
\hline P3 & 5 & $\begin{array}{c}\text { NAL-AMP- } \\
\text { CFM/CTX-TET- } \\
\text { SXT }\end{array}$ & 12 & $\begin{array}{c}\text { Schwarzengrund } \\
(10) \\
\text { Typhimurium (1) } \\
\text { Heidelberg (1) }\end{array}$ & $\begin{array}{l}\text { Heces }(8)^{\mathrm{H}} \\
\text { Pollo }(4)^{\mathrm{A}}\end{array}$ \\
\hline P4 & 4 & $\begin{array}{c}\text { AMP-CHL-NIT- } \\
\text { TET }\end{array}$ & 7 & Infantis (7) & Heces $(7)^{H}$ \\
\hline P5 & 4 & $\begin{array}{c}\text { AMP-CFM/CTX- } \\
\text { TET-SXT }\end{array}$ & 6 & $\begin{array}{c}\text { Schwarzengrund } \\
(6)\end{array}$ & $\begin{array}{l}\text { Heces (5) }{ }^{\mathrm{H}} \\
\text { Bovino (1) }{ }^{\mathrm{A}}\end{array}$ \\
\hline P6 & 3 & AMP-GEN-TET & 6 & Typhimurium (6) & $\begin{array}{l}\text { Heces (5) }{ }^{\mathrm{H}} \\
\text { Sangre (1) }{ }^{\mathrm{H}}\end{array}$ \\
\hline P7 & 5 & $\begin{array}{c}\text { NAL/CIP-AMC- } \\
\text { AMP-CFM/CTX- } \\
\text { TET }\end{array}$ & 5 & $\begin{array}{l}\text { Heidelberg (4) } \\
\text { S. enterica (1) }\end{array}$ & $\begin{array}{c}\text { Pollo (5) } \\
\text { Balanceados (1) }\end{array}$ \\
\hline P8 & 3 & $\begin{array}{c}\text { NAL/CIP-NIT- } \\
\text { TET }\end{array}$ & 4 & $\begin{array}{l}\text { Heidelberg (2) } \\
\text { Javiana (1) } \\
\text { S. enterica (1) }\end{array}$ & $\begin{array}{c}\text { Pollo (3) } \\
\text { Balanceados (1) }\end{array}$ \\
\hline P9 & 6 & $\begin{array}{c}\text { NAL-AMC- } \\
\text { AMP-CFM/CTX- } \\
\text { NIT-TET }\end{array}$ & 4 & $\begin{array}{l}\text { Heidelberg (2) } \\
\text { S. enterica (2) }\end{array}$ & $\begin{array}{l}\text { Pollo (3) }{ }^{\mathrm{A}} \\
\text { Bovino (1) }\end{array}$ \\
\hline P10 & 5 & $\begin{array}{l}\text { NAL/CIP-AMP- } \\
\text { CHL-NIT-TET }\end{array}$ & 3 & $\begin{array}{l}\text { Typhimurium (2) } \\
\text { S. enterica (1) }\end{array}$ & Heces $(3)^{\mathrm{H}}$ \\
\hline P11 & 4 & $\begin{array}{l}\text { NAL/CIP-AMP- } \\
\text { GEN-TET }\end{array}$ & 4 & Typhimurium (4) & $\begin{array}{l}\text { Heces }(3)^{\mathrm{H}} \\
\text { Sangre (1) }\end{array}$ \\
\hline P12 & 4 & $\begin{array}{l}\text { NAL-AMP-CHL- } \\
\text { TET }\end{array}$ & 3 & Typhimurium (3) & Heces $(3)^{H}$ \\
\hline P13 & 3 & AMP-CHL-TET & 3 & Infantis (2) & Heces (2) ${ }^{\mathrm{H}}$ \\
\hline
\end{tabular}




\begin{tabular}{|c|c|c|c|c|c|}
\hline & & & & Zaiman (1) & Porcino $(1)^{A}$ \\
\hline P14 & 3 & NAL-GEN-TET & 2 & Heidelberg (2) & Pollo(2) ${ }^{A}$ \\
\hline P15 & 4 & $\begin{array}{l}\text { AMP-CFM/CTX- } \\
\text { GEN-TET }\end{array}$ & 2 & Typhimurium (2) & Heces $(2)^{H}$ \\
\hline PX & $3--6$ & $\begin{array}{c}\text { Otros } \\
\text { fenotipos }\end{array}$ & $\begin{array}{c}22 \\
1 \mathrm{c} / \mathrm{u}\end{array}$ & $\begin{array}{c}\text { Typhimurium } \\
(10) \\
\text { Schwarzengrund } \\
(3) \\
\text { Enteritidis (2) } \\
\text { Oranienburg (2) } \\
\text { Heidelberg (1) } \\
\text { Newport (1) } \\
\text { Cerro (1) } \\
\text { Havana (1) } \\
\text { S. enterica (1) }\end{array}$ & $\begin{array}{c}\text { Heces }(16)^{\mathrm{H}} \\
\text { Sangre }(1)^{\mathrm{H}} \\
\text { Orina }(1)^{\mathrm{H}} \\
\text { Balanceados }(2)^{\mathrm{A}} \\
\text { Pollo }(1)^{\mathrm{A}} \\
\text { Porcino }(1)^{\mathrm{A}}\end{array}$ \\
\hline
\end{tabular}

*MDR: Aislamiento bacteriano resistente a por lo menos un fármaco de tres o más familias o grupos de antimicrobianos incluidos en el estudio. En el caso de las Quinolonas la resistencia simultánea a Ac. Nalidixico NAL(1 $1^{\circ}$ Generación) y Ciprofloxacina CIP ( $2^{\circ}$ Generación), se considera como una sola. \#A: muestra de alimentos de origen animal \# H: muestra clínica de origen humano.

El serotipo Heidelberg representó el 38\% de estas cepas (34/113), Typhimurium $31 \%(28 / 113)$ y Schwarzengrund $21 \%(19 / 113)$; el pollo fue el tipo de alimento predominante $(46 / 113)$ y en su mayoría las muestras humanas fueron heces (55/113).

\section{DISCUSIÓN}

En el presente trabajo se describe la situación epidemiológica en nuestro país, de la resistencia antimicrobiana y prevalencia de serovariedades de Salmonella enterica aisladas a partir de muestras clínicas humanas y de alimentos durante tres años consecutivos.

Los serotipos encontrados fueron de gran diversidad en las diferentes muestras estudiadas, con prevalencia de $S$. serovar Heidelberg en alimentos y $S$. serovar Typhimurium en humanos.

Se observó resistencia antimicrobiana en $35.6 \%$ de los aislamientos, los antibióticos con mayor resistencia fueron Tetraciclina, Ác. Nalidíxico, Ampicilina y Nitrofurantoína, con algunos cambios de tendencia al considerar cada grupo de forma independiente, siendo Ampicilina el segundo más resistente en aislamientos de humanos. La resistencia simultánea a múltiples fármacos MDR se evidenció en el $16,9 \%$ de los aislamientos.

En ambas poblaciones, aunque ligeramente mayor en alimentos se observaron cepas resistentes a cefalosporinas de $3^{a}$ generación (C $3^{a} G$ ) y a ciprofloxacina, fármacos comúnmente utilizados en el tratamiento de infecciones humanas; además un $27,5 \%$ mostró valores de sensibilidad intermedios para ciprofloxacina lo cual podría traducirse en la posibilidad de fracasos terapéuticos durante su uso. La resistencia a $C 3^{a} \mathrm{G}$ estuvo dada por mecanismos transferibles por plásmidos como betalactamasas de espectro extendido BLEE y AmpC plasmídico, lo que permitiría mayor diseminación de la resistencia.

En un estudio previo realizado en nuestro país ${ }^{(29)}$ sobre Salmonella spp aisladas de diferentes fuentes durante los años 2011-2012, se reportó elevada resistencia para Nitrofurantoína en cepas de muestras humanas, de alimentos y animales; a diferencia de los datos actuales, se observó resistencia a Tetraciclina sólo en alimentos y escasa - nula resistencia a $\beta$-lactámicos y Ciprofloxacina, lo que refleja un incremento importante en la resistencia de Salmonella a nivel local.

Un hallazgo interesante encontrado en alimentos de origen aviar, fue la prevalencia del serovar Heidelberg con la betalactamasa de tipo AmpC plasmídico como mecanismo más frecuente de resistencia a C3 ${ }^{a} \mathrm{G}$. Este paralelismo serotipo/resistencia ya fue descrito por Tijerino et al en Costa $\operatorname{Rica}^{(30)}$ y en un trabajo publicado en Holanda por vanden Berg RR et $\mathrm{al}^{(31)}$ donde aislaron este serovar de muestras de pollo 
de origen sudamericano, presentando el mismo mecanismo de resistencia en alto porcentaje.

En relación con la resistencia a múltiples fármacos en los aislamientos de origen humano se encontró que el 16,2\% (58/357) fueron Salmonella MDR con 27 patrones de resistencia diferentes. Estos resultados son ligeramente más altos comparados con Frasson et al $^{(18)}$ de Italia donde reportaron $13,4 \%$ de MDR, pero coinciden con nuestros datos en el predominio del serovar Typhimurium y la alta resistencia a Tetraciclina y Ampicilina. Se observan cifras superiores a las nuestras en otra publicación de Willi Quino et al $^{(32)}$ en Perú donde estudiaron cepas de origen humano del serovar Infantis reportando $74,1 \%$ de MDR con 13 patrones de resistencia; con respecto a este serovar observamos un aumento de casos en el año 2019 en nuestro país.

Los aislamientos provenientes de alimentos presentaron un $17,7 \%$ (55/311) de MDR con 13 patrones de resistencia, estos resultados son mayores comparados con el estudio de Voss Rech et $\mathrm{al}^{(33)}$ en Brasil donde reportaron 9,5\% de Salmonella MDR en aves, con 15 serovares presentes entre ellos Heidelberg; sin embargo en otro estudio Villagómez et $\mathrm{al}^{\left({ }^{(34)}\right.}$ en Ecuador encontraron cifras muy superiores con $87,8 \%$ de MDR y 12 patrones de resistencia en cepas aisladas en una empresa avícola donde el serovar Infantis fue el predominante, serotipo que no fue aislado a partir de alimentos en el presente trabajo. Estudios realizados en otras fuentes de alimentos como en México por Ballesteros et al $^{(35)}$ encontraron $73,7 \%$ de MDR con 11 patrones de resistencia en Salmonella aisladas en carne de res, y en Colombia Bermúdez et al ${ }^{(12)}$ reportaron $98,6 \%$ aislamientos MDR con 52 patrones de resistencia en plantas de beneficio porcino.

Los resultados encontrados constituyen un valioso aporte al conocimiento de la epidemiología de Salmonella en Paraguay, evidenciando la circulación de gran diversidad de serotipos tanto en muestras de humanos como en alimentos de origen animal, así como un crecido porcentaje de aislamientos resistentes $(35,6 \%)$ a los antibióticos vigilados y la presencia no despreciable de cepas MDR, muchas de las cuales afectan a los antimicrobianos utilizados para el tratamiento de infecciones humanas.

Los serotipos de Salmonella asociados a mayor resistencia a los antibióticos son Heidelberg relacionados a alimentos de origen aviar, Typhimurium a muestras de infecciones gastrointestinales y Schwarzengrund presente tanto en alimentos como en muestras clínicas humanas.

La resistencia bacteriana es un fenómeno global y por supuesto nuestro país está afectado, cada vez se reportan más casos de multirresistencia en bacilos gram negativos ${ }^{(36,37)}$ y Salmonella no es la excepción ${ }^{(38-40)}$. Este aumento está ligado al uso indiscriminado de antibióticos en la agricultura, ganadería y ambiente hospitalario. El impacto es alto en la salud humana y la economía, por ello es fundamental seguir fortaleciendo la vigilancia del avance de la resistencia de este grupo bacteriano en todos los sectores mencionados para implementar estrategias de control y mejorar las que ya existen.

\section{Conflicto de interés}

El presente trabajo no presenta conflictos de interés.

\section{Aspectos éticos}

Se cuenta con Dictamen de aprobación del Comité de Ética de Investigación del Laboratorio Central de Salud Pública. 


\section{REFERENCIAS BIBLIOGRAFICAS}

1. Organización Mundial de la Salud. Estimaciones de la OMS de la carga mundial de enfermedades transmitidas por los alimentos: grupo de referencia de epidemiología de la carga de enfermedades transmitidas por los alimentos 2007-2015.OMS. 2015,https://apps.who.int/iris/handle/ $10665 / 199350$

2. CDC.2018. Surveillance for Foodborne Disease Outbreaks -United States, 2009-2015

3. Su L-H, Chiu C-H, Chu C, Ou JT. Antimicrobial resistance in Nontyphoid Salmonella Serotypes: A Global Challenge. Clinical infectious Disease, 39(4), 15 August 2004,pages 546-551 $10.1086 / 422726$

4. Junod T, López-Martín J, Gädicke P. Estudio de susceptibilidad antimicrobiana de Salmonella enterica en muestras de origen animal $y$ alimentario. Rev. méd. Chile (Internet). 2013 Mar (citado 2020 Jun 24); 141(3): 298-304. Disponible en: https://scielo.conicyt.cl/scielo.php?scri $\mathrm{pt}=\mathrm{sci}$ arttext\&pid=S0034-

$98872013000300003 \&$ Ing $=$ es. 10.4067/S0034-8872013000300003.

5. Andino A and Hanning I. Salmonella enterica: Survival, Colonization, and Virulence Differences among Serovars. The Scientific World Journal. 2015. 116. $10.1155 / 2015 / 520179$

6. Quesada A, Reginita GA, Ruiz Español A, Colantonio LD, Burrone MS. Resistencia Antimicrobiana de Salmonella spp aislada de alimentos de origen animal para consumo humano. Rev Perú Med Exp Salud Publica. 2016;33(1):32-44

7. Iramain $R$, Jara $A$, Martínez Tovilla $Y$, Cardozo L, Morínigo R, Rojas P, et al. Consenso Internacional de Gastroenteritis Aguda en Urgencias. Comité de Emergencias SLACIP. Pediatr. (Asunción) 2017; 44(3):24958.

8. Yalda LA. Etiología y manejo de la gastroenteritis aguda infecciosa en niños y adultos. Rev Med Clin Condes. 2014; 25 (3): 463 - 472. Doi: 10.1016/S0716-8640(14) 70063-X.

9. Rivera Calderón LG, Motta Delgado PA, Cerón Urbano MF, Chimonja Coy FA. Resistencia de la Salmonela a los antimicrobianos convencionales para su tratamiento. Revista CES Medicina Veterinaria y Zootecnia [Internet]. 2012;7(1):116-129. Recuperado de: https://www.redalyc.org/articulo.oa?id $=3214281070$
10. Barreto M, Castillo-Ruiz M, Retamal P. Salmonella enterica: una revisión de la trilogía agente, hospedero y ambiente, y su trascendencia en Chile. Rev chilena Infectol. 2016; 33 (5): 547-557

11. Bermúdez PM, Pulecio SL, Suárez MC. Susceptibilidad Antimicrobiana de Aislamientos de Salmonella enterica provenientes de pisos, equipos, utensilios y producto terminado en plantas de beneficio porcino en Colombia. Revista de la Facultad de Medicina Veterinaria y de Zootecnia (Internet). 2016; 63(1):39-53. Recuperado de: https://www.redalyc.org/articulo.oa?id $=407645831005$

12. Alós JI. Resistencia bacteriana a los antibióticos: una crisis global. Enferm Infecc Microbiol Clin. 2015; 33(10) :692-99. 10.1016/j.eimc.2014.10.004

13. Organización Mundial de la Salud. (2014) Resistencia a los antimicrobianos: informe global sobre vigilancia. Organización Mundial de la Salud.

https://apps.who.int/iris/handle/1066 5/112642

14. Organización Panamericana de la Salud / Organización Mundial de la Salud. Alerta Epidemiológica: Enterobacterias con resistencia transferible a colistina, implicaciones para la salud publica en las Américas, 10 de junio de 2016, Washington, D.C. OPS/OMS. 2016

15. Organización Panamericana de la Salud / Organización Mundial de la Salud. Alerta Epidemiológica: Salmonella entérica serovar Typhi haplotipo H58. 10 de octubre de 2018, Washington, D.C. OPS/OMS. 2018.

16. World Health Organization. Prioritization of pathogens to guide discovery, research and development of new antibiotics for drug-resistant bacterial infections, including tuberculosis. World Health Organization Geneva; 2017(WHO/EMP/IAU/2017.12).

(License: CC BY-NC-SA 3.0 IGO.

17. Wongsuvan $G$, Wuthiekanun $V$, Hinjoy S, Día NP, Limmathurotsakul D. Uso de antibióticos en aves de corral: una encuesta de ocho granjas en Tailandia. Bull Organo Mundial de la Salud. 2018; 96 (2): 94-100. doi: 10.2471 / BLT.17.195834

18. Frasson I, Bettanello S, De Canals E, Richter SN, Palù G. Serotype epidemiology and multidrug resistance patterns of Salmonella enterica 
infecting humans in Italy. Gut Pathog. 2016; 8:26. DOI $10.1186 /$ s13099016-0110-8

19. Zhang $L$, Fu Y, Xiong Z, Ma Y, Wei Y, Qu $X$, et al. Highly Prevalent Multidrug-Resistant Salmonella from Chicken and Pork Meat at Retail Markets in Guangdong, China. Front. Microbiol.2018 9: 2104. doi: 10.3389/fmicb.2018.02104

20. Cuong N, Padungtod P, Thwaites G, Carrique-Mas J. Uso de antimicrobianos en la producción animal: una revisión de la literatura con un enfoque en países de bajos y medianos ingresos. Antibióticos (Internet) 2018; 7: 75. Disponible en: 10.3390/antibiotics7030075

21. Del Pozo Liliana, Nazario Silva, Valencia Augusto, Soto Javier, Riveros Juan C, Sacsaquispe Rosa et al. Estudio de un brote intrahospitalario por Salmonella typhimurium productora de beta-lactamasa de espectro extendido SHV-5. An. Fac. med. [Internet]. 2006 oct (citado 2020 Ago 19); 67(4): 318$326 . \quad$ Disponible en: http://www.scielo.org.pe/scielo.php?s cript $=$ sci_arttext\&pid=S1025$55832006000400006 \&$ lng =es.

22. Miro E, Vergés C, García I, Mirelis B, Navarro F, Coll P, et al. Resistencia a quinolonas y betalactámicos en Salmonella enterica, y su relación con mutaciones en las topoisomerasas, alteraciones en la permeabilidad celular y expresión de un mecanismo de expulsión activa. Enferm Inflexo Microbiol Clin. 2004; 22(4):204-11

23. VT Nair D, Venkitanarayanan K, Kollanoor Johny A. Salmonella resistente a los antibióticos en el suministro de alimentos y el papel potencial de las alternativas antibióticas para el control. Alimentos (Internet). MDPI AG; 11 de octubre de 2018; 7 (10): 167. Disponible en: 10.3390/foods7100167.

24. Jiménez Pearson MA, Galas $M$, Corso A, Hormazábal JC, Duarte Valderrama $\mathrm{C}$, Salgado Marcano $\mathrm{N}$ et al. Consenso latinoamericano para definir, categorizar y notificar patógenos multirresistentes, con resistencia extendida 0 panresistentes. Rev Panam Salud Publica. 2019; 43:65. 10.26633/RPSP.2019.65

25. Wolfensberger, A., Kuster, SP, Marchesi, M. et al. El efecto de las diferentes definiciones de resistencia a múltiples fármacos (MDR) sobre las tasas de bacilos gramnegativos MDR.
Antimicrob Resist Infect Control 8, 193 (2019). 10.1186/s13756-019-0614-3

26. Magiorakos AP, Srinivasan A, Carey RB, Carmeli Y, Falagas ME, Giske CG, et al. Multidrug-resistant, extensively drug-resistant and pandrug-resistant bacteria: an international expert proposal for interim standard definitions for acquired resistance. Clin Microbiol Infect. 2012; 18:268-81. DOI: $10.1111 / j .1469-$ 0691.2011.03570.x

27. German GJ, Jamieson FB, Gilmour $M$, Almohri $H$, Bullard J, Domingo MC, et al. Interim Recommendations for the Reporting of Extensively Drug Resistant and Pan Drug Resistant Isolates of Enterobacteriaceae, Pseudomonas aeruginosa, Acinetobacter spp. and Stenotrophomonas maltophilia. Can Commun Dis Rep. 7 de abril de 2016;42(4):96-104. 10.14745/ccdr.v42i04a04

28. German GJ, Gilmour M, Tipples G, Adam HJ, Almohri $\mathrm{H}$, Bullard J, et al. Canadian recommendations for laboratory interpretation of multiple or extensive drug resistance in clinical isolates of Enterobacteriaceae, Acinetobacter species and Pseudomonas aeruginosa. Can Commun Dis Rep. 2018;44(1):29-34. 10.14745/ccdr. v44i01a07

29. Weiler N, Orrego M, Álvarez M, Huber $C$, Ortiz $F$, Núñez $L$, Piris $L$, Pérez $J$. Primeros resultados de la vigilancia integrada de la resistencia antimicrobiana de patógenos transmitidos por alimentos, Campylobacter spp. y Salmonella spp. en tres poblaciones distintas. Paraguay. 2011-2012. Mem. Inst. Investig. Cienc. Salud. 2017; 15(2): 64-72

30. Tijerino Ayala A, Bolaños Acuña HM, Acuña Calvo MT, Vargas Morales JL, Campos Chacón E. Emergencia de $\beta$ lactamasa AmpC plasmídica del grupo CMY-2 en Shigella sonnei y Salmonella spp. en Costa Rica, 2003-2015. Rev Panam Salud Publica. 2016;40(1):705.

31. van den Berg RR, Dissel $S$, Rapallini MLBA, van der Weijden CC, Wit B, Heymans R. Characterization and whole genome sequencing of closely related multidrug-resistant Salmonella enterica serovar Heidelberg isolates from imported poultry meat in the Netherlands. Polos One. 2019; 14(7): e0219795. Published 2019 Jul 22. doi: 10.1371/journal.pone.0219795 
32. Quino W, Hurtado CV, EscalanteMaldonado $O$, Flores-León $D$, Mestanza O, Vences-Rosales $\mathrm{F}$, et al. Multidrogorresistencia de Salmonella Infantis en Perú: un estudio mediante secuenciamiento de nueva generación. Rev Perú Med Exp Salud Publica. 2019;36(1): 3745.doi: $10.17843 /$ rpmesp. 2019.361.39 34.

33. Voss-Rech $D$, Vaz CSL, Alves $L$, Coldebella A, Leão JA, Rodrigues DP, et al. A temporal study of Salmonella enterica serotypes from broiler farms in Brazil. Poultry Science. marzo de 2015;94(3):433-41.

$10.3382 / \mathrm{ps} /$ peu081

34. Villagómez Estrada S, Logacho Pilataxi M, Vinueza Burgos C. Presencia y Resistencia a los Antimicrobianos de serovariedades de Salmonella enterica en una empresa avícola integrada del Ecuador. Rev Ecuat Med Cienc Biol. 2017; 38 (1):11-25.DOI:10.26807/ remcb. v38i1.17

35. Nayarit-Ballesteros N, Rubio-Lozano MS, Delgado-Suárez E, MéndezMedina D, Braña-Varela D, RodasSuárez O. Perfil de resistencia a antibióticos de serotipos de Salmonella spp. aislados de carne de res molida en la Ciudad de México. Salud pública Méx (revista en la Internet). 2016 jun (citado 2020Jul08); 58(3):371-377. Disponible en: http://www.scielo.org.mx/scielo.php?s cript $=$ sci_arttext\&pid $=$ S0036$36342016000300371 \&$ Ing $=$ es. 10.21149/spm.v58i3.7897.

36. Melgarejo N, Martínez M., Franco R., Falcón M. Enterobacterias resistentes a Carbapenemes por producción de KPC, aisladas en hospitales de
Asunción y Departamento Central. Rev. Salud Pública Parag. 2013; 3 (1): 30-5

37. Nancy Melgarejo Touchet, Mario Martínez, Rossana Franco, Miryan Falcón, Sofia Busignani, Carmen Espínola et al. Resistencia plasmídica a colistin por el gen mcr-1 en Enterobacteriaceae en Paraguay. Rev. salud publica Parag. (Internet). 2018 June

(cited 2021 Apr 26); 8(1):44-48.

Available from:

http://scielo.iics.una.py/scielo.php?scr ipt $=$ sci_arttext $\&$ pid $=$ S2307-

33492018000100044\&lng=en. 10.18 004/rspp.2018.junio.44-48.

38. Melgarejo-T Nancy, Martínez Mario, Franco Rossana, Falcón Miryan, Álvarez Mercedes, Ortiz Helena et al. Primer aislamiento de Salmonella Javiana con portación de KPC-2 en Paraguay. Rev. salud publica Parag. (Internet). $2017 \quad$ Dic (citado 2021 Abr 26); 7(2): 51-56. Disponible en: http://scielo.iics.una.py/scielo.php?scr ipt $=$ sci_arttext\&pid=S2307$33492017000200051 \&$ Ing $=$ es. 10.18004/rspp.2017.diciembre.51-56.

39. Lima T, Domingues S, Da Silva G. Resistencia a la colistina mediada por plásmidos en Salmonella enterica: una revisión. Microorganismos [Internet]. MDPI AG; 201919 de febrero; 7 (2): $55 . \quad$ Disponible en: 10.3390/microorganisms7020055

40. Cortés-Sánchez, J, García-Barrientos A., Minor-Pérez, R., et al. A.L.S. (2017) Food Safety and Antimicrobial Resistance an Approach to the Genus Salmonella spp. Journal of Biosciences and Medicines, 5, 55-71. 10.4236/jbm.2017.52006 